\title{
Visualizing Elements in the Shafarevich-Tate Group
}

\author{
John E. Cremona and Barry Mazur
}

To Bryan Birch

\section{CONTENTS}

\section{Introduction}

1. Elements of $\amalg(E / K)$ Represented as Étale Coverings of $E$

2. Elements of $\amalg(E / K)$ Represented as Curves of Degree $n$ in Projective $(n-1)$-Space

3. Elements of $\amalg(E / K)$ Represented as Curves in Abelian Varieties

4. Experimental Data

5. Asymptotic Questions

Acknowledgements

References
We review a number of ways of "visualizing" the elements of the Shafarevich-Tate group of an elliptic curve E over a number field $\mathrm{K}$. We are specifically interested in cases where the elliptic curves are defined over the rationals, and are subabelian varieties of the new part of the jacobian of a modular curve (specifically, of $X_{0}(N)$, where $N$ is the conductor of the elliptic curve). For a given such $\mathrm{E}$ with nontrivial Shafarevich-Tate group, we pose the question:

Are all the curves of genus one representing elements of the Shafarevich-Tate group of E isomorphic (over the rationals) to curves contained in a (single) abelian surface $A$, itself defined over the rationals, containing $E$ as a subelliptic curve, and contained in turn in the new part of the jacobian of a modular curve $\mathrm{X}_{0}(\mathrm{~N})$ ?

At first view, one might imagine that there are few $\mathrm{E}$ with nontrivial Shafarevich-Tate group for which the answer is yes. Indeed we have a small number of examples where the answer is no, and it is very likely that the answer will be no if the order of the Shafarevich-Tate group is large enough. Nonetheless, among all (modular) elliptic curves $\mathrm{E}$ as above, with conductors up to 5500 and with no rational point of order 2, we have found the answer to the question to be yes in the vast majority of cases. We are puzzled by this and wonder whether there is some conceptual reason for it. We present a substantial amount of data relating to the curves investigated.

\section{INTRODUCTION}

Two basic arithmetic invariants of an elliptic curve $E$ over a number field $K$ are

- the Mordell-Weil group $E(K)$, whose elements are the $K$-rational points of $E$, and

- the Shafarevich-Tate group $\amalg(E / K)$, whose elements are defined to be isomorphism classes of pairs $(T, \iota)$ where $T$ is a smooth projective curve of genus 1 over $K$ possessing a $K_{v}$-rational point

(C) A K Peters, Ltd. 1058-6458/2000 $\$ 0.50$ per page Experimental Mathematics 9:1, page 13 
for every place $v$ of $K$ (where $K_{v}$ is the completion of $K$ at $v$ ), and where

$$
\iota: E \rightarrow \mathrm{jac} T
$$

is an isomorphism over $K$ between $E$ and the jacobian of $T$.

As is well known, $E(K)$ and $\amalg(E / K)$ are somehow linked in the sense that it is often easier to come by information about the Selmer group of $E$ over $K$ which is built out of both $E(K)$ and $\amalg(E / K)$ than it is to get information about either of these groups separately. It occurred to us that, although these two groups (Mordell-Weil and $\amalg$ ) are partners, so to speak, in the arithmetic analysis of the elliptic curve $E$, there seems to be a slight discrepancy in their treatment in the existent mathematical literature, for this literature does a much more thorough job of helping one (at least in specific instances) to compute rational points, i.e., to exhibit elements of Mordell-Weil, than it does in helping one to find (in an explicit way) the curves of genus one which represent elements of $\amalg$ (especially if one is interested in elements of $\amalg$ of order greater than 2). This is perhaps understandable in that it is usually quite clear how to present a rational point (e.g., if $E$ is given in Weierstrass form, giving just its $x$-coordinate determines the rational point up to sign) but it is less clear what manner one should choose to exhibit the curves of genus 1 representing the elements of $\amalg$. Of course (for a fixed integer $n$ ) an element in $\amalg$ annihilated by multiplication by $n$ can always be obtained by push-out, starting with an appropriate 1cocycle on the Galois group $G_{K}=\operatorname{Gal}(\bar{K} / K)$ with coefficients in the finite Galois module $E[n] \subset E$, the kernel of multiplication by $n$ in $E$, (the 1-cocycle being unramified outside the primes dividing $n$ and the places of bad reduction for $E$ ) and so therefore, there is indeed, a "finitistic" way of representing these elements of $\amalg$. Our aim here is rather to develop strategies that might enable us to "visualize" the underlying curves more concretely. There are, for example, two standard ways of representing elements of $\amalg$, both of which we will briefly review below, and we will also suggest a third (where the curves of genus 1 in question are sought as subcurves of abelian varieties). It is this third mode of visualizing elements of the Shafarevich-Tate group together with data regarding it (see Tables 1 and 2 on pages 25 and 26) that is the principal theme of our article.

The data we tabulate strike us as surprising, and as deserving of some explanation. However, we have no hypothesis to offer that would explain it, and therefore our article is not genuinely experimental in the classical sense (despite the name of the journal in which it appears), since experiments are usually expected to be the testing-grounds of explicitly articulated hypotheses.

Explicit equations for curves of genus 1 and for their jacobians, together with results regarding visibility and related matters, were the subject of a Winter School at the University of Arizona in March 1999. See http://www.math.arizona.edu/ swcenter/ aws99/ for more details.

\section{ELEMENTS OF Ш(E/K) REPRESENTED AS ÉTALE COVERINGS OF $\mathrm{E}$}

Let $n$ be a positive integer. Given $T$ a curve of genus 1 over $K$ with a specific identification of its jacobian with $E$, there is a natural action of $E$ on $T$ which allows us to view $T$ as a principal homogeneous space (equivalent terminology: torsor) for $E$ over $K$. If $T$ represents an element of order $n$ in $\amalg(E / K)$ (or more generally, an element of the "Weil-Châtelet" group $\mathrm{WC}(E / K) \cong H^{1}\left(G_{K}, E\right)$, of isomorphism classes of $E$-torsors over $K$ ) the quotient of $T$ under the action of the finite subgroup $E[n] \subset E$ has a $K$-rational point, and is therefore $K$-isomorphic to $E$. That is, we may view $T$ as an étale finite covering of $E$, of degree $n^{2}$.

\section{ELEMENTS OF Ш(E/K) REPRESENTED AS CURVES OF DEGREE $n$ IN PROJECTIVE $(n-1)$-SPACE}

Now let us give ourselves $T$, a curve of genus 1 over $K$, with an identification of its jacobian with $E$, representing an element $\sigma$ of order $n>1$ in $\amalg(E / K)$, and note that for any integer $k \in \mathbb{Z}$ the curve $T^{k}:=\operatorname{Pic}^{k}(T)$ of linear equivalence classes of divisors of degree $k$ on $T$ is again a torsor for $E$ over $K$ representing the element $k \cdot \sigma \in \amalg(E / K)$. In particular, since $T^{n} \cong E$ (over $K$ ) we see that there exists a linear equivalence class of divisors of degree $n$ on $T$ which is $K$-rational. Choose such a $K$-rational 
divisor class $\mathcal{D}$, and consider the (Chow) variety $V$ (over $K$ ) consisting of divisors on $T$ which are in the linear equivalence class $\mathcal{D}$. Over $\bar{K}$ the variety $V$ is a projective space, and $V$ is therefore a (BrauerSeveri) twist of projective space over $K$. But since $\sigma \in \amalg(E / K)$, it follows that $V$ has a $K_{v}$-rational point for all completions $K_{v}$ of $K$ and therefore, by Global Class Field Theory (more specifically, by the Hasse Principle for Brauer-Severi varieties) $V$ has a $K$-rational point; i.e., there is a $K$-rational divisor on $T$ of degree $n$. Choose such a divisor $D$, and consider the mapping (of degree $n$ ) $r_{D}$ of $T$ to the $(n-1)$-dimensional projective space

$$
\mathbb{P}^{n-1}:=\mathbb{P}\left(H^{0}(T, \mathcal{O}(D))\right),
$$

defined over $K$ by the linear system of $D$. This representation of $T$ is independent of the rational divisor $D$ chosen, in the sense that given another choice, $D^{\prime}$, the representation $r_{D^{\prime}}$ may be obtained from $r_{D}$ by composition of appropriate $K$-isomorphisms of domain and range. We might remark that this method of representing elements of $\amalg$, in contrast with the first method we described, works as formulated specifically for elements of the ShafarevichTate group but if one were to try to extend it to a method of describing curves $T$ representing elements of order $n$ in the larger Weil-Châtelet group one would be required, in general, to replace the ambient projective $(n-1)$-space by an appropriate Brauer-Severi variety of dimension $n-1$ over $K$.

Returning to the case at hand, i.e., representing elements of $\amalg$, when $n=2$ the above method represents $T$ as double cover of $\mathbb{P}^{1}$. When $n \geq 3$ we get $T$ as a curve, defined over $K$, of degree $n$ in $\mathbb{P}^{n-1}$. In particular, when $n=3, T$ is represented, in this way, as a plane cubic. There is a large body of classical literature (but, nevertheless, many still-open problems) regarding this case and the case $n=4$; we will review some of this literature below. When $n=4, T$ is represented as a curve of degree 4 in $\mathbb{P}^{3}$ which is also the subject of significant classical work (the legacy of Jacobi). Also in more recent times, the legacy of Jacobi has been expressed in terms of the theory of theta functions via the Heisenberg representation [Mumford 1966]. If appropriately developed, this approach might yield, we believe, a fine format for presenting the equations of curves of degree $n$ in $\mathbb{P}^{n-1}$ representing elements of $\amalg$.

\section{The Case $\mathrm{n}=3$}

By the height of a plane cubic over $K$ (i.e., a cubic in the standard projective plane, given with homogeneous coordinates $X_{0}, X_{1}, X_{2}$ ) let us mean the logarithmic height of the point in projective 9 -space of the (ten) homogeneous coordinates of the defining equation of the cubic. To get a notion of height that is independent of the coordinatization of the projective plane, call the minimal height of a plane cubic over $K$ the greatest lower bound of these heights under projective general linear changes of the homogeneous coordinates $X_{0}, X_{1}, X_{2}$ defined over $K$; to actually compute this minimal height would involve understanding the classical reduction theory regarding the symmetric cube representation of $\mathrm{GL}_{3}$, and implementing algorithms for it. But given this, we have a well-defined notion of the minimal height $h(\sigma)$ of an element $\sigma$ of order 3 in $\amalg(E / K)$ : one defines $h(\sigma)$ to be the minimal height of a plane cubic representing $\sigma$.

Problem. When $K=\mathbb{Q}$, find an upper bound as a function of $N=\operatorname{conductor}(E)$ for the minimal heights of all elements of order 3 in $\amalg(E / \mathbb{Q})$.

\section{Some Literature and Current Work on the Explicit Representation of Curves of Genus 1 and Their Jacobians}

In search of explicit formulas, there are two directions in which it is important to go. One can start with a curve of genus 1, given by an equation, or a system of equations, and ask for the equation(s) of its jacobian. Or, and this is the more specific thrust of this article, one can try go the other way: given an elliptic curve, and a Selmer class, find the explicit equations of the curve of genus 1 representing that class. There is a wealth of material which goes in the first direction (e.g., typical of such is the result of Cassels about plane diagonal cubics: for nonzero constants $a, b, c$ in a field of characteristic different from 3 , the plane cubic curve whose equation is $a X^{3}+b Y^{3}+c Z^{3}=0$ has jacobian isomorphic to the locus of zeroes of $X^{3}+Y^{3}+a b c Z^{3}$ ). For the jacobian of curves of genus 1 where the curves are of order $n$ in their Weil-Châtelet groups and for the equations of the $n$-fold map to the jacobian, see [Weil 1954] or [Cremona 2000] for $n=2$, [Salmon 1879] for $n=3$, and, when $n=4$ and we have given the curve in question as an intersection of two 
quadrics in $\mathbb{P}^{3}$, see [Salmon 1928] or [Merriman et al. 1996]. For the formulas for the jacobians of curves of genus 1 given as hypersurfaces of bihomogenous degree $(2,2)$ in $\mathbb{P}^{1} \times \mathbb{P}^{1}$ see the Harvard Ph.D. thesis of Catherine O'Neil [1999], who has found families $\mathcal{C}_{2}$, $\mathcal{C}_{3}$, and $\mathcal{C}_{5}$ of curves of genus one in $\mathbb{P}^{1} \times \mathbb{P}^{1}, \mathbb{P}^{2}$, and $\mathbb{P}^{4}$ respectively such that $(1) \mathrm{A}$ map $\mathcal{C}_{i} \longrightarrow$ jac $\left(\mathcal{C}_{i}\right)$ is explicitly written as a linear automorphism of the ambient projective space, and (2) every curve of genus one over a field $F$ of characteristic 0 embeddable over $F$ in one of the projective or multiprojective spaces above, and whose jacobian has a subgroup of $i$-torsion isomorphic (over $F$ ) to $\mu_{i}$ is a member of $\mathcal{C}_{i}$.

The general formula in the cases $n \leq 4$ is the subject of a paper [An et al. 1999] being presently written by McCallum, Minhyong Kim and some of the graduate students at the University of Arizona (Sang Yook An, Susan Hammond, Seog Young Kim, David Marshall, and Alex Perlis).

For $n=5$, as Nicholas Shepherd-Barron pointed out to one of us, the equations for a smooth curve of genus 1 of degree 5 in $\mathbb{P}^{4}$ can be given as the determinants of minors of a $5 \times 5$ Pfaffian matrix. The search for elliptic curves over $\mathbb{Q}$ with large 5Selmer group is the subject of current work being done by Tom Fisher, a student of Shepherd-Barron, who does this by writing down genus 1 curves of degree 5 in $\mathbb{P}^{4}$, with an action of $\mu_{5}$, the corresponding jacobians being isogenous to the quotients of these by $\mu_{5}$ [Fisher 2000].

There are fewer results of an explicit nature going "the other way". Available numerical data (such as listings of equations of minimal height representing the elements of order 3 in the Shafarevich-Tate groups of elliptic curves of low conductor) are still fragmentary at best.

\section{ELEMENTS OF Ш(E/K) REPRESENTED AS CURVES IN ABELIAN VARIETIES}

Let $\sigma$ be an element in $\mathrm{WC}(E / K) \cong H^{1}\left(G_{K}, E\right)$, the Weil-Châtelet group of isomorphism classes of torsors for $E$ over $K$. Suppose that we are given an embedding over $K$ of $E$ into an abelian variety $J$. Form the exact sequence of abelian varieties

$$
0 \rightarrow E \rightarrow J \rightarrow B \rightarrow 0
$$

Definition. We say that $\sigma$ is visible in $J$ if $\sigma$ is in the kernel of the natural homomorphism

$$
\mathrm{WC}(E / K) \rightarrow \mathrm{WC}(J / K) .
$$

Remark 1. The element $\sigma$ is visible in $J$ if and only if there is an element $\beta \in B(K)$ such that $\sigma$ is represented by a curve $T$ of genus 1 defined over $K$ contained in the variety $J$ and such that $T$ is the inverse image of the point $\beta \in B$ under the projection $J \rightarrow B$. Equivalently, $T$ is a translation of $E$ by a point $P \in J(\bar{K})$, the point $P$ projecting to $\beta$ under the natural mapping $J \rightarrow B$. Thus

$$
T:=E+P \subset J .
$$

(Of course, if $\sigma \neq 0$, the point $P$ is not rational over $K$ despite the fact that the translate $E+P$ is defined over $K$.)

Proof. This follows immediately upon consideration of the exact sequence $(*)$ and the induced long exact sequence of $G_{K^{-}}$cohomology:

$$
J(K) \rightarrow B(K) \rightarrow H^{1}\left(G_{K}, E\right) \rightarrow H^{1}\left(G_{K}, J\right) .
$$

Definition. If the situation above occurs, we shall say that the element $\sigma$ is explained by the element $\beta \in B(K)$ of the Mordell-Weil group of $B$, noting that the element $\beta$ playing the role required in the statement of the theorem is uniquely determined modulo the image of $J(K)$ in $B(K)$.

Since the curve $T$ representing $\sigma$ is the inverse image of an element $\beta \in B(K)$ explaining $\sigma$, the size of the coefficients of the equations for $T$, as, say, a curve in some projective space, is bounded by data coming from a choice of projective embedding of $J$, the nature of the projection mapping $J \rightarrow B$, and, finally, the height of the point $\beta$.

Remark 2. Suppose that our elliptic curve $E$ does not have complex multiplication by $\sqrt{-1}$ or $\sqrt{-3}$, and we have an embedding of $E$ into an abelian variety $J$ (over $K$ ) such that there are no nontrivial homomorphisms of $E$ to $B=J / E$ over $\bar{K}$. Then an element $\sigma \in \mathrm{WC}(E / K)$ is visible in $J$ if and only if the curve $T$ of genus 1 (over $K$ ) representing $\sigma$ is isomorphic over $K$ to a curve contained in the variety $J$.

Proof. By Remark 1, if $\sigma$ is visible in $J$, then $T$ occurs as a subvariety (in fact, it is a translate of $E$ ) in $J$. 
Suppose that $T$ is isomorphic to a subvariety $T^{\prime} \subset$ $J$. The projection $J \rightarrow B$ must be constant when restricted to $T^{\prime}$, for $T^{\prime}$ is isomorphic over $\bar{K}$ to $E$ and, by assumption, there are no nonconstant maps from $E$ to $B$ over $\bar{K}$. So $T^{\prime}$ is a translate of $E$. We must show that the structure that $T^{\prime}$ inherits from $T$ as torsor over $E$ coincides, up to sign, with the $E$-torsor structure on $T^{\prime}$ given by addition (in $J$ ). But by our assumption on $E$, we have that the only automorphisms of $E$ are the scalar multiplications by \pm 1 , and therefore, up to sign, there is only one $E$-torsor structure on $T^{\prime}$, which concludes the proof of this remark.

Remark 3. As Johan de Jong explained to one of us (in the Castle pub on Castle Hill in Cambridge, England), for any element $\sigma \in \mathrm{WC}(E / K)$ there is some abelian variety $J$ over $K$ containing $E$ as abelian subvariety, such that $\sigma$ is visible in $J$. One can see this as follows. Let $n$ be the order of $\sigma$, and represent $\sigma$ as an Azumaya algebra $\mathcal{A}_{F}$ of rank $n^{2}$ over the field $F$ of rational functions on the $K$-variety $E$. There is a maximal commutative sub-algebra $L$ in $\mathcal{A}$ of rank $n$ over $F$ such that, if $\pi: C \rightarrow E$ is the mapping of degree $n$ of projective smooth curves associated to the field extension $L / F$, then $\pi$ is totally ramified at (at least) one point of $E$. It follows that the associated morphism of jacobians $E=J_{E} \rightarrow J_{C}$ is injective. Moreover, by construction, the induced Azumaya algebra $\mathcal{A}_{L}=\mathcal{A}_{F} \otimes_{F} L$ splits; i.e. $\sigma$ is visible in $J_{C}$. Here are the details:

Proposition. Let $K$ be a number field, $E$ an elliptic curve over $K$ and $\sigma \in \mathrm{WC}(E / K)$. Then there is some abelian variety $J$ over $K$ containing $E$ as abelian subvariety, such that $\sigma$ is visible in $J$.

Proof. Consider the natural homomorphism

$$
H^{1}(K, E) \rightarrow \coprod_{v} H^{1}\left(K_{v}, E\right)
$$

where $v$ runs through all non-archimedean places of $K$, and where $K_{v}$ is the completion of $K$ at $v$. Let $\mathcal{V}$ denote the finite set of these places which have the property that the element $\sigma \in H^{1}(K, E)$ does not go to zero under the mapping $H^{1}(K, E) \rightarrow H^{1}\left(K_{v}, E\right)$. To have a nice geometric model to work with, let $\mathcal{O}=\mathcal{O}_{K}[1 / m] \subset K$ be a Dedekind subdomain of the ring of integers $\mathcal{O}_{K}$ of $K$ where we have inverted the non-zero integer $m$; the integer $m$ is assumed to be divisible by all primes of bad reduction for $E$ and by the residual characteristics of all $v \in \mathcal{V}$ and by the order of $\sigma$. It follows that the cohomology class $\sigma$ comes by restriction from a class (which we denote by the same letter) $\sigma \in H^{1}(\operatorname{Spec} \mathcal{O}, \mathcal{E})$, where $f: \mathcal{E} \rightarrow \operatorname{Spec} \mathcal{O}$ is the Néron model of $E / K$ over the base Spec $\mathcal{O}$, and the cohomology in question is étale cohomology. Alternatively, we may view $\sigma$ as an element of the kernel of

$$
H^{1}(K, E) \rightarrow \coprod_{v \notin \mathcal{V}} H^{1}\left(K_{v}, E\right) ;
$$

i.e., the group denoted $\amalg(V, A)$ in [Tate 1968, Section 3] for $V=\mathcal{O}$ and $A=\mathcal{E}$. We may apply [Tate 1968, Theorem 3.1] to the proper morphism $f: \mathcal{E} \rightarrow \operatorname{Spec} \mathcal{O}$ (its fibers are of dimension 1 and $\mathcal{E}$ is regular of dimension 2) to get the exact sequence

$$
0 \rightarrow \operatorname{Br}(\operatorname{Spec}(\mathcal{O})) \rightarrow \operatorname{Br}(\mathcal{E}) \rightarrow \amalg(\mathcal{O}, \mathcal{E}) \rightarrow 0 .
$$

By surjectivity of $\operatorname{Br}(\mathcal{E}) \rightarrow \amalg(\mathcal{E})$, we may (and do) choose an element $\xi$ in the Brauer group of $\mathcal{E}$ which projects to $\sigma$. We now "shrink" $\operatorname{Spec}(\mathcal{O})$ further, so as to guarantee that the order (call it $N$ ) of the element $\xi$ is not divisible by any of the residual characteristics of $\operatorname{Spec}(\mathcal{O})$, and therefore $\xi$ is the image of some element $\eta \in H^{2}\left(\mathcal{E}, \mu_{N}\right)$ under the mapping

$$
H^{2}\left(\mathcal{\varepsilon}, \mu_{N}\right) \rightarrow H^{2}\left(\mathcal{E}, \boldsymbol{G}_{m}\right)=\operatorname{Br}(\mathcal{E}) .
$$

We now modify our choice of lifting $\xi$. Let $\hat{\mathcal{E}}$ denote the completion of (the abelian scheme) $\mathcal{E}$ along its zero-section, and let

$$
z: \operatorname{Spec}(\mathcal{O}) \hookrightarrow \hat{\mathcal{E}}
$$

denote that zero-section. Let $\hat{\eta} \in H^{2}\left(\hat{\mathcal{E}}, \mu_{N}\right)$ be the pullback of the cohomology class $\eta$ to $\hat{\mathcal{E}}$. The morphism $z$ above induces an isomorphism on étale cohomology,

$$
z: H^{2}\left(\hat{\mathcal{E}}, \mu_{N}\right) \cong H^{2}\left(\operatorname{Spec}(\mathcal{O}), \mu_{N}\right),
$$

and let let us denote by $\eta_{\circ} \in H^{2}\left(\operatorname{Spec}(\mathcal{O}), \mu_{N}\right)$ the image of $\hat{\eta}$ under the isomorphism $z$. Let $\xi_{\circ} \in$ $H^{2}\left(\operatorname{Spec}(\mathcal{O}), \boldsymbol{G}_{m}\right)=\operatorname{Br}(\operatorname{Spec}(\mathcal{O}))$ be the image of $\eta_{\circ}$ under the mapping

$$
H^{2}\left(\operatorname{Spec}(\mathcal{O}), \mu_{N}\right) \rightarrow H^{2}\left(\operatorname{Spec}(\mathcal{O}), \boldsymbol{G}_{m}\right) .
$$

Put

$$
\xi^{\prime}:=\xi \text { - image of } \xi_{\circ} \text { in } \operatorname{Br}(\mathcal{E}) .
$$

Then $\xi^{\prime}$ is also a lifting of $\sigma$, but has the added property that its pullback to $\operatorname{Br}(\hat{\mathcal{E}})$ vanishes. Let $n$ 
denote its order, and let $\mathcal{A}_{\varepsilon}$ denote an Azumaya algebra of rank $n^{2}$ over $\mathcal{E}$ representing $\xi^{\prime}$. Such an Azumaya algebra exists by [Grothendieck 1968, Corollary 2.2]. Moreover, the Azumaya algebra $\mathcal{A}_{\hat{\varepsilon}}$ is a "trivial" Azumaya algebra over $\hat{\mathcal{E}}$.

We now retract to the associated function fields: let $F$ denote the field of rational functions on the $K$-variety $E$ which we view as a discretely valued field, with the valuation given by the order of zero (or pole) at the origin of the elliptic curve $E$. Let $F_{\circ}$ denote the completion of $F$ with respect to this valuation. Thus, $F_{\circ} \cong K((t))$ is isomorphic to the field of Laurent power series in a uniformizer $t$. Let $\mathcal{A}_{F}$ be the central simple algebra (of rank $n^{2}$ ) over $F$ which is obtained by change of scalars from the Azumaya algebra $\mathcal{A}_{\hat{\varepsilon}}$. The central simple algebra $\mathcal{A}_{F_{\circ}}$ obtained from $\mathcal{A}_{F}$ by base change is trivial; that is, it is a total matrix algebra $\operatorname{Mat}_{n}\left(F_{\circ}\right)$ of all $n \times n$ matrices with entries in $\left.F_{\circ} \cong K((t))\right)$. Here is how we may view this total matrix algebra. Identifying $F_{\circ}$ with $K((t))$, let $L_{\circ} / F_{\circ}$ be the totally ramified extension of degree $n$ given by $L_{\circ}:=K((s))$ where $s^{n}=t$; i.e., $L_{\circ}:=K\left(\left(t^{1 / n}\right)\right)$. Viewing $L_{\circ}$ as $(n$ dimensional) vector space over $F_{\circ}$, we may find an isomorphism, then, between $F_{\circ}$-algebras:

$$
\mathcal{A}_{F_{\circ}} \cong \operatorname{End}_{F_{\circ}}\left(L_{\circ}\right) \cong \operatorname{Mat}_{n}\left(F_{\circ}\right)
$$

and since $L_{\circ}$ is a maximal commutative algebra (of rank $n)$ in $\operatorname{End}_{F_{\circ}}\left(L_{\circ}\right)$, its action on the $F_{\circ}$-vector space given by multiplication, so we have an imbedding of $L_{\circ}$ into $\mathcal{A}_{F_{\circ}}$.

Our next task is to approximate the uniformizer

$$
s \in L_{\circ} \subset \mathcal{A}_{F_{\circ}}
$$

by an element $s^{\prime} \in \mathcal{A}_{F}$. Since $\mathcal{A}_{F}$ is dense in the topological vector space $\mathcal{A}_{F_{0}}$, given any positive integer $\nu$, we can find such an element $s^{\prime}$ with the property that

$$
s^{\prime}-s=t^{\nu} \cdot w \in \mathcal{A}_{F_{\mathrm{o}}} \cong \operatorname{Mat}_{n}(K((t))),
$$

where $w \in \operatorname{Mat}_{n}(K[[t]])$. If $\nu$ is taken large enough, we get that the characteristic polynomial for the action of $s^{\prime}$ is a monic polynomial of degree $n$ which is congruent modulo a high power of $t$ to the polynomial $X^{n}-t$, and therefore $s^{\prime}$ generates a maximal commutative subfield $L$ of $\mathcal{A}_{F}$ (an extension of $F$ of degree $n$ ) which is totally ramified over $F_{\circ}$.
We now have only to repeat the brief sketch given immediately before the statement of this proposition. Namely, let $C$ be the smooth projective curve whose field of rational functions is $L$ (i.e., the normalization of $L$ over the $K$-scheme $E$ ) and note that since the natural projection mapping $C \rightarrow E$ is totally ramified at the origin in $E$, it induces an injection on jacobians $0 \rightarrow E \rightarrow J:=\operatorname{jac}(C)$ and, moreover we see, by the construction of $C$, that the Azumaya algebra $\mathcal{A}$ splits when pulled back to $C$. That is, $\sigma$ is visible in $J=\operatorname{jac} C$.

This construction, however, does not allow us easy viewing of the curves of genus 1 that are generated. To get a sharper image we are led to imposing very strong restrictions on the types of abelian varieties $J$ that we wish to use, to visualize torsors over elliptic curves. For the rest of this article, we concentrate in the question of visualizing elements of $\amalg$ rather than the corresponding more general question for arbitrary $E$-torsors. Moreover, we will be interested in five special situations.

1. The field $K=\mathbb{Q}$, the elliptic curve $E$ a abelian subvariety of $J_{0}(N):=\operatorname{jac}\left(X_{0}(N)\right)$ the jacobian of the modular curve $X_{0}(N)$ for some level $N$, and we want to know which elements of $\amalg(E / \mathbb{Q})$ are visible in $J_{0}(N)$.

2. We are over any number field $K$ and we want the elements of $\amalg$ visible in abelian surfaces.

3. Same as item 1 above, but considering only elliptic curves $E \subset J_{0}(N)$ where $N$ is specifically the conductor of $E$, and we want to know the elements of $\amalg$ visible in $J_{0}(N)$.

4. The combination of items $1,2,3$ above. That is, we are over $K=\mathbb{Q}$ and are seeking elements of $\amalg$ visible in abelian surfaces contained in $J_{0}(N)$ where $N$ is the conductor of $E$.

5. As in item 4 above, but with one more specific requirement. As in item 4 , we are dealing with elliptic curves $E$ over $K=\mathbb{Q}$ and are seeking elements of $\amalg(E / \mathbb{Q})$ visible in abelian surfaces $J$,

$$
E \subset J \subset J_{0}(N)
$$

where $N$ is the conductor of $E$, but also request that the complementary elliptic curve $A \subset J$ to $E$ in the abelian surface $J$ be of conductor $N$ as well (equivalently: that $J$ be contained in the new part of $\left.J_{0}(N)\right)$. 
You might imagine that we are stacking the deck against ourselves by asking for something as stringent as item 5 , but we are getting ahead of our story.

\section{Visibility and Congruence Moduli}

Let $0 \rightarrow E \rightarrow J \rightarrow B \rightarrow 0$ be an exact sequence of abelian varieties over $K$, where $E$ is an elliptic curve. Denote by $A \subset J$ a complementary abelian variety to $E$ in $J$, so that we have the exact sequence over $K$,

$$
0 \rightarrow A \cap E \rightarrow A \oplus E \rightarrow J \rightarrow 0,
$$

with $A \cap E$ a finite subgroup of the abelian varieties $A$ and $E$; we embed it "anti-" diagonally in $A \oplus E$. Let $m$ be the exponent of the finite group

$$
(A \cap E)(\bar{K}) .
$$

We can call the integer $m$ the congruence modulus of $E$ and $A$ in $J$. One immediately sees that if $\sigma \in$ $\amalg(E / K)$ is visible in $J$ then its order divides the congruence modulus $m$, and, more specifically, there is an element $h \in H^{1}\left(G_{K}, E \cap A\right)$ that maps to

$$
\sigma \in \amalg(E / K) \subset H^{1}\left(G_{K}, E\right)
$$

under the homomorphism induced from the inclusion $E \cap A \hookrightarrow E$, and to $0 \in H^{1}\left(G_{K}, A\right)$ under the homomorphism induced from the inclusion $E \cap A \hookrightarrow$ $A$. The set of elements of $\amalg(E / K)$ visible in $J$ is a subgroup of $\amalg(E / K)$, and is a subgroup of $\amalg(E / K)[m]$. Denote the subgroup of elements of $\amalg(E / K)$ visible in $J$ by

$$
\amalg(E / K)^{(J)} \subset \amalg(E / K)[m] \subset \amalg(E / K) .
$$

There is a converse to this description. We give ourselves the following data:

(i) an abelian variety $A$ over $K$,

(ii) finite, $G_{K^{-}}$-stable, subgroups $\Phi_{E} \subset E$ and $\Phi_{A} \subset$ $A$, and

(iii) a $G_{K}$-equivariant isomorphism $\iota: \Phi_{E} \cong \Phi_{A}$,

these data satisfying these properties:

(a) $\sigma \in \amalg(E / K) \subset H^{1}\left(G_{K}, E\right)$ is the image of an element $h \in H^{1}\left(G_{K}, \Phi_{E}\right)$;

(b) $\iota \cdot h \in H^{1}\left(G_{K}, \Phi_{A}\right)$ maps to zero in $H^{1}\left(G_{K}, A\right)$ under the homomorphism induced from the inclusion $\Phi_{A} \hookrightarrow A$.
Then, forming $J$ by requiring the sequence

$$
0 \rightarrow \Phi_{E} \rightarrow A \oplus E \rightarrow J \rightarrow 0
$$

to be exact, where we have embedded

$$
\Phi_{E} \hookrightarrow A \oplus E
$$

by the injection $\iota \oplus-1$, the element $\sigma$ is visible in $J, A$ is a complementary abelian variety to $E$ in $J$, and the congruence modulus is the exponent of the finite group $\Phi_{E} \cong \Phi_{A}$.

Referring to our list of cases above, Case 2 occurs when the abelian variety $A$ is an elliptic curve. Note, therefore, that one would expect there to be serious impediments to finding visible elements of U of large order (for fixed $K$ ) in abelian surfaces. For example we would not even expect to find pairs of non-isogenous elliptic curves $E, A$ over $\mathbb{Q}$ with $\mathbb{Q}$ stable finite subgroups $\Phi_{E} \subset E$ and $\Phi_{A} \subset A$ which are $G_{\mathbb{Q}^{-}}$equivariantly isomorphic and are of large exponent $m$ (let alone with the properties requisite for visibility).

Specifically, one of us (Cremona) has conducted a search for non-isogenous pairs of elliptic curves $E$ and $A$ for which there are finite subgroups $\Phi_{E} \subset E$ and $\Phi_{A} \subset A$ which are $G_{\mathbb{Q}^{-}}$-equivariantly isomorphic of exponent $m$. This search has so far covered all (modular) elliptic curves of conductor $N \leq 5500$ and all prime moduli $m \leq 97$. It has yielded a large number of examples for $m \leq 7$, quite a number for $m=11$, but has so far yielded only two examples for $m \geq 13$, both of these being for $m=$ 13. Namely, there is an elliptic curve of conductor 988, labelled 988B1 in [Cremona 1997], satisfying a 13-congruence (see below for the definition of $m$-congruence) with the elliptic curve $52 \mathrm{~A} 1$ of conductor 52; and the elliptic curve $3952 \mathrm{C} 1$ satisfies a 13-congruence with the curve $208 \mathrm{C} 1$. Neither of these congruences involve issues of visibility. These curves all have trivial $\amalg$ and rank 0, except for 988B1 which has rank 1.

This systematic search shows that there are no $m$-congruences for pairs of non-isogenous (modular) elliptic curves of conductors both $\leq 5500$, where $m$ is a prime number in the range $17 \leq m \leq 97$. The question of "high congruences" satisfied by pairs of non-isogenous elliptic curves is a topic of some current interest. See, for example, [Kani and Schanz 1997; 1998; Carlton 1998]. 


\section{Optimal (or "Strong Weil") Modular Elliptic Curves}

A natural case to consider is where $K=\mathbb{Q}$, and $E$ is a modular elliptic curve over $\mathbb{Q}$ of conductor $N$, contained in the jacobian of the modular curve $J=J_{0}(N):=\operatorname{jac}\left(X_{0}(N)\right)$. The requirement that $E$ be contained in $J_{0}(N)$ is, in effect, the requirement that $E$ be the optimal (or equivalently, in somewhat older terminology, the "strong Weil") elliptic curve in its $\mathbb{Q}$-isogeny class. It is equivalent to request that the modular parametrization

$$
\pi: X_{0}(N) \rightarrow E
$$

of smallest degree among all possible nonconstant mappings from $X_{0}(N)$ to $E$ have the property that the kernel of the homomorphism induced from $\pi$ on jacobians, $J_{0}(N) \rightarrow E$, be (geometrically) irreducible. By definition, the modular degree of $E$, denoted $m_{E}$, is the degree of the finite mapping $\pi$. Denoting its kernel $A \subset J:=J_{0}(N)$, we have that $A$ is an abelian variety over $K$ which fits into the exact sequence

$$
0 \rightarrow A \rightarrow J \rightarrow E \rightarrow 0
$$

whose dual we identify with

$$
0 \rightarrow E \rightarrow J \rightarrow B \rightarrow 0 .
$$

The appropriate compositions of the mappings in the exact sequences above give us isogenies $E \rightarrow E$ and $A \rightarrow B$, the first being multiplication by the modular degree, $m_{E}$, from which we deduce that the (common) kernel of these isogenies is the finite subgroup $A \cap E=E\left[m_{E}\right]$. In particular, the congruence modulus of $E$ and $A$ in $J$ is equal to the modular degree of $E$.

In studying the Shafarevich-Tate groups of elliptic curves, the optimal curve is a good choice of curve to concentrate on, in that, at least as far as most of the available numerical data shows, the order of the Shafarevich-Tate group, if it varies at all within a given isogeny class, will tend to be smallest for the optimal curve in the class. The phrase "will tend" is perhaps a bit too weak to describe the state of affairs here: of the data so far analyzed by the first author (going up to level 1000), there are only two counter-examples, both at level 960 , to the statement that the minimal order of the ShafarevichTate group is attained by the optimal member of the $\mathbb{Q}$-isogeny class of modular elliptic curves. The exceptions are the isogeny classes 960D and 960N (in the labelling of [Cremona 1997]), where the optimal curves $960 \mathrm{D} 1$ and $960 \mathrm{~N} 1$ both have $\amalg$ of order 4 , while in each case the three other curves in the isogeny class have trivial Ш. (See [Cremona 1993] for more details of this investigation.)

It would be interesting to determine whether these counter-examples remain "optimal" when considered as quotients of $X_{1}(N)$, following the ideas regarding optimality suggested in [Stevens 1989]. We have not yet answered this question, but we suspect that the answer in each case is "yes", for the following reason. Stevens proves in [Stevens 1989] that each isogeny class of elliptic curves of conductor $N$ over $\mathbb{Q}$ contains a unique curve whose Faltings-Parshin height is minimal, or equivalently whose period lattice is strictly contained in the period lattices of the other curves in the class. He also conjectures that the curve of minimal height is always the $X_{1}(N)$ optimal curve in the class, and proves (by explicit computation) that this holds for $N \leq 200$. For both the classes $960 \mathrm{D}$ and $960 \mathrm{~N}$, the $X_{0}(N)$-optimal curves have minimal height, so by Stevens' conjecture one would expect that they are also $X_{1}(N)$ optimal.

In any event, once one knows the ShafarevichTate group of one member of a $\mathbb{Q}$-isogeny class of elliptic curves, it is often not that hard to work out the Shafarevich-Tate group of any other member. In the above situation, denoting as above by $B$ the quotient abelian variety $J / E$, we have most of the hypothesis requested in Remark 2 above (that there are no nontrivial homomorphisms from $E$ to $B$ ) by the "multiplicity one" theorem.

Denote the subgroup of elements of the Shafarevich-Tate group of a modular elliptic curve $E$ of conductor $N$ which are visible in the modular jacobian $J=J_{0}(N)$ with a superscript ${ }^{\circ}$, so we have the inclusion of subgroups

$$
\amalg(E / \mathbb{Q})^{\circ} \subset \amalg(E / \mathbb{Q})\left[m_{E}\right] \subset \amalg(E / \mathbb{Q}),
$$

and note also the evident fact that whenever the modular degree of $E$ is prime to the order of the torsion group of $B(K)$, any

$$
\sigma \in \amalg(E / \mathbb{Q})^{\circ}
$$

is "explained by" an element $\beta \in B(K)$ of infinite order. 


\section{The Relation of $\mathrm{m}$-Congruence}

Let $E$ and $F$ be elliptic curves over a field $K$, and let $m>0$ be a positive integer. We will say that $E$ and $F$ are $m$-congruent over $K$ if there exists an isomorphism $E[m] \cong F[m]$ as $(\mathbb{Z} / m \mathbb{Z})\left[G_{K}\right]$-modules. Suppose $E$ and $F$, now, are optimal elliptic curves over $\mathbb{Q}$ of the same conductor $N$ and denote by

$$
f_{E}(q)=q+a_{2}(E) q^{2}+a_{3}(E) q^{3}+\cdots
$$

the Fourier expansion of the cuspidal modular newform of weight two on $\Gamma_{0}(N)$ corresponding to $E$, and by $f_{F}(q)$ the Fourier expansion of the newform corresponding to $F$. The newforms $f_{E}$ and $f_{F}$ are eigenforms for the full Hecke algebra $\mathbb{T}=\mathbb{T}_{0}(N)$ which acts faithfully on the space of cuspidal modular forms of weight two on $\Gamma_{0}(N)$ (and also on the jacobian, $J_{0}(N)$, of the modular curve $X_{0}(N)$ ) and which is generated by the $T_{l}$ 's for prime numbers $l$ not dividing the level $N$ together with the $U_{q}$ 's for primes $q$ dividing $N$. Our elliptic curves $E$ and $F$ are both abelian subvarieties of the new part of $J_{0}(N)$. To simplify our discussion, suppose that $m=p$ is a prime number. Consider these five conditions.

(1) The "prime to $p N$ " Fourier coefficients of $f_{E}$ and $f_{F}$ "satisfy a $p$-congruence", i.e., $a_{n}(E) \equiv a_{n}(F)$ $\bmod p$ for all $n$ such that $(n, p N)=1$.

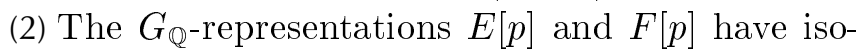
morphic semisimplifications.

(3) The $G_{\mathbb{Q}^{-}}$-representations $E[p]$ and $F[p]$ are isomorphic (equivalently, $E$ and $F$ are $p$-congruent).

(4) All the Fourier coefficients $a_{n}(E) \equiv a_{n}(F) \bmod p$ for all $n$ (" $p$-congruence").

(5) The finite subgroups $E[p]$ and $F[p]$ are equal in $J_{0}(N)$. That is, the abelian subvarieties $E \subset$ $J_{0}(N)$ and $F \subset J_{0}(N)$ have the property that their intersection contains $E[p]=F[p]$.

There are some evident implications between these five conditions. But also, (1) and (2) are equivalent, and when the Galois representation $E[p]$ is irreducible (or, what amounts to the same thing, when $E$ does not admit a rational $p$-isogeny) (1), (2), and (3) are equivalent. Moreover, if $N$ is relatively prime to $p, p$ is odd, and $E[p]$ irreducible, then (4) and (5) are equivalent (by [Ribet 1990, Theorem 5.2]). We also have the equivalence of (4) and (5) when $p$ divides $N$ provided that $p$ is odd, $p^{2}$ doesn't divide $N$, and the Galois representation on $E[p]$ is irreducible and not finite at $p$ [Mazur and Ribet 1991]. The condition that $E$ be not finite at $p$ is equivalent, if $p^{2}$ does not divide $N$, to the requirement that $\operatorname{ord}_{p}\left(\Delta_{E}\right)$ not be congruent to zero modulo $p$, where $\Delta_{E}$ is the discriminant of $E$.

We will refer to condition (5) as providing a modular p-congruence between $E$ and $F$. So, we have (at least) two possible notions: modular p-congruence, and (the a priori weaker notion of) p-congruence.

There are two possible computational strategies for checking, for a given positive integer $m$, that $E[m]=F[m]$ (e.g., when $m=p$ is a prime number, for checking a "modular $p$-congruence").

First strategy: Computing m-congruences of period lattices. The better of the two ways is to explicitly determine a basis for the integral homology of $E$ and of $F$ in $H_{1}\left(X_{0}(N) ; \mathbb{Z}\right)$, and then to demonstrate that corresponding basis elements are linearly dependent modulo $m$. This has the virtue of actually demonstrating that $E[m]=F[m]$. It is by this method that we establish most of the modular $p$-congruences listed in our table, using the modular symbol methods of [Cremona 1997].

Second strategy: Computing congruences of Fourier coefficients, and order of vanishing of $\Delta$. Another possible computational strategy to establish modular $p$ congruences is suggested by the following proposition, whose proof follows from the results already quoted in [Ribet 1990; Mazur and Ribet 1991].

Proposition. Let $N$ be an integer, and $p$ an odd prime number such that $p^{2}$ does not divide $N$. Let $E$ and $F$ be elliptic curves defined over $\mathbb{Q}$ both (of conductor $N$, and) contained as abelian subvarieties of the new part of $J_{0}(N)$. Suppose that the $G_{\mathbb{Q}}$-representation on $E[p]$ is irreducible.

Then $E[p]=F[p]$ as subgroups of $J_{0}(N)$ (and, in particular, conditions (1)-(5) all hold) if

(i) $a_{n}(E) \equiv a_{n}(F) \bmod p$ for all $n$, and

(ii) if $p$ divides $N, \operatorname{ord}_{p}\left(\Delta_{E}\right)$ is not congruent to 0 $\bmod p$.

To implement this strategy for $m=p$, we must check (i) and (ii). Of course, (ii) only requires a finite number of different computations and therefore it is feasible, and very easy in the cases of interest to us, to make such a check. But (i) involves an 
infinite number of distinct computations. Here we make the following convention: if we have checked that $a_{l}(E) \equiv a_{l}(F) \bmod p$ for all prime numbers $l<1000$, and if, in the few cases where there are prime divisors $l$ of $p N$ which are greater than 1000, we also have checked the $p$-congruence for these $l$ 's as well, we will say that the pair $E$ and $F$ seem to satisfy a p-congruence. If, further, the hypotheses of the proposition, together with (ii) also hold, we will then also say that such a pair $E$ and $F$ seem to satisfy a modular p-congruence. In any such instance, if one wanted to actually prove the existence of a $p$ congruence or modular $p$-congruence, further work would be necessary: for example, one could use the results of [Sturm 1987] to reduce the checking of (i) to the checking of a finite number of congruences.

However, as we have mentioned, for most of the cases tabulated below (including all those in Table 1 , where $m$ is odd) we have been able to follow the first strategy and therefore we will have shown that the congruence $a_{n}(E) \equiv a_{n}(F) \bmod m$ does in fact hold for all $n$. When we have only established that a $p$-congruence, or modular $p$-congruence, seems to be the case we explicitly indicate this in the tables.

Remark. Assume the Birch-Swinnerton-Dyer Conjecture and the Shafarevich-Tate Conjecture. If $E$ and $F$ are optimal, of the same conductor $N$, and are modular $p$-congruent one to another $(p>2)$ then the parity of the Mordell-Weil ranks of $E$ and $F$ are the same.

To see this, just note that the parity of the MordellWeil ranks is determined by the sign of the eigenvalue \pm 1 of the operator $w_{N}$ on $E$ and $F$ as they sit in $J_{0}(N)$, and since $p>2$ this sign can be read off by the action of $w_{N}$ on $E[p]=F[p]$.

\section{EXPERIMENTAL DATA}

\section{The First Two Examples}

It may very well be that "asymptotically" for high values of the conductor $N$, the subgroup $\amalg(E / K)^{\circ}$ of visible elements does not account for a large portion of $\amalg(E / K)$ or even of $\amalg(E / K)\left[m_{E}\right]$. Nonetheless, we began to examine the issue by considering the "first" two instances of nontrivial ShafarevichTate group for optimal semi-stable elliptic curves (i.e. the two lowest conductors $N$ for which this occurs). These are tabulated in [Cremona 1997] and are the curves labelled 571A1 and $681 \mathrm{~B} 1$ there. The curve 571A1 has trivial Mordell-Weil group, and the Mordell-Weil group of 681B1 consists of 2torsion; their Shafarevich-Tate groups are isomorphic to $\mathbb{Z} / 2 \mathbb{Z} \times \mathbb{Z} / 2 \mathbb{Z}$ and to $\mathbb{Z} / 3 \mathbb{Z} \times \mathbb{Z} / 3 \mathbb{Z}$, respectively. Checking [Cremona 1997] one immediately finds the happy "accident" that 571A1 admits a 2-congruence with the optimal elliptic curve factor 571B1, whose Mordell-Weil rank is 2 and whose 2-part of $\mathrm{II}$ is trivial. And with $681 \mathrm{~B} 1$, a similar "accident" happens: 681B1 seems to admit a 3-congruence with the optimal elliptic curve factor 681C1, whose Mordell-Weil rank is 2 and whose 3 part of $\amalg$ is trivial. Further computation, using the "first strategy" given above, shows that these congruences do hold fully in both cases, in the sense that condition (5) of the previous page holds: the 2-torsion of 571A1 and 571B1 coincide in $J_{0}(571)$, and the 3 -torsion of $681 \mathrm{~B} 1$ and $681 \mathrm{C} 1$ coincide in $J_{0}(681)$.

The values of the orders of $\amalg$ given in [Cremona 1997] and [Cremona 1993] are in all cases the socalled "analytic order" of $\amalg$, which is the order as predicted from the value of the $L$-series at $s=1$ by the conjecture of Birch and Swinnerton-Dyer; hence these data and the data that will be tabulated below should be taken as conditional on this conjecture.

We therefore officially assume the truth of the Birch and Swinnerton-Dyer conjecture for the rest of this article.

It follows that all of $\amalg(571 \mathrm{~A} 1 / \mathbb{Q})$ is visible in the abelian surface $J:=(571 \mathrm{~A} 1 \oplus 571 \mathrm{~B} 1) / \Phi$, where $\Phi$ is isomorphic to the kernel of multiplication by 2 in either $571 \mathrm{~A} 1$ or $571 \mathrm{~B} 1$, and is embedded diagonally. Moreover, the two independent generators of the Mordell-Weil group of 571B1 explain the two independent generators modulo 2 of $\amalg(571 \mathrm{~A} 1 / \mathbb{Q})$. Similarly, all of $\amalg(681 \mathrm{~B} 1 / \mathbb{Q})$ is visible in the abelian surface $J:=(681 \mathrm{~B} 1 \oplus 681 \mathrm{C} 1) / \Phi$ where $\Phi$ is isomorphic to the kernel of multiplication by 3 in either $681 \mathrm{~B} 1$ or $681 \mathrm{C} 1$, and, again, the two independent generators of the Mordell-Weil group of $681 \mathrm{C} 1$ explain the two independent generators modulo 3 of $\amalg(681 B 1 / \mathbb{Q})$. Moreover the abelian surface $J=(681 \mathrm{~B} 1 \oplus 681 \mathrm{C} 1) / \Phi$ is an abelian subvariety of $J_{0}(681)$. 


\section{About the Data}

To make some further tests to see whether these were two extremely lucky, but singular, occurrences, Adam Logan examined squarefree conductors $N<$ 3000 with the help of data and programs of the first author [Cremona 1999]. Logan showed that all elements of odd order in the Shafarevich-Tate groups of optimal semi-stable elliptic curves over $\mathbb{Q}$ of conductor $N<2849$ are visible in abelian surfaces contained in the jacobian $J_{0}(N)$ (these computations being again conditional upon the conjecture of Birch and Swinnerton-Dyer, and on the assumption that certain "apparent" $m$-congruences are actual $m$-congruences). In this regard, one should also mention the surprising computations done by Amod Agashé [1999] (a Ph.D. student of Loïc Merel) who, along with Merel, has been independently investigating the order of the Shafarevich-Tate group of the winding quotients of $J_{0}(N)$ for $N$ prime. They find that $\amalg\left(J_{0}(N)\right)$ vanishes surprisingly often (but not always; for example, there is an element of order 7 in $\left.\amalg\left(J_{0}(1091)\right)\right)$.

The first author has since continued Logan's investigation to all levels up to 5500 . In the rest of this paper we will present and discuss the data obtained.

\section{The Data in Detail}

It appears that all of the elliptic curves with nontrivial Shafarevich-Tate group with conductor at most 5500 have Mordell-Weil rank 0. Two caveats are necessary here, however: first, we have not yet made systematic tables of the (analytic) order of $\amalg$ for non-optimal curves in the higher range $1000<N \leq$ 5500 which was not already covered in [Cremona 1993]. Second, for optimal curves of positive rank $r$, our claim that the analytic order of $\amalg$ is trivial is based upon the assumption that the $r$ independent points we have (as listed in [Cremona 1997] and supplementary computer files in [Cremona 1999]) do generate the full Mordell-Weil group modulo torsion, rather than a subgroup of index greater than 1. We have only checked this in some cases.

The nontrivial Shafarevich-Tate groups for $N$ in this range are either of order $p^{2}$ for $p=2,3,5$ or 7 or else of order 16 . Specifically, there are 153 occurrences of order 4,37 of order 9,11 of order 16 , 13 of order 25 and one of order 49 . In discussing the data, it is useful to distinguish between instances where the Shafarevich-Tate group is of odd order or of order a power of two, these being the only cases that arise in the range tabulated. We remark that for all the cases where $\amalg$ has order 16, a 2-descent using mwrank [Cremona 1998] shows that the 2-rank of $\amalg$ is 2 .

\section{The Kernel of Multiplication by the Modular Degree}

Recall the inclusion of subgroups of the ShafarevichTate group of $E$,

$$
\amalg(E / \mathbb{Q})^{\circ} \subset \amalg(E / \mathbb{Q})\left[m_{E}\right] \subset \amalg(E / \mathbb{Q}) .
$$

We find only three cases, where the modular degree $m_{E}$ does not annihilate all of $\amalg(E / \mathbb{Q})$, i.e., where $\amalg(E / \mathbb{Q})\left[m_{E}\right]$ differs from $\amalg(E / \mathbb{Q})$. The first, found by Logan, is given by the curve $E=$ 2849A1 which has $\amalg(E / \mathbb{Q})$ of order 9 , but modular degree not divisible by 3 . In particular, none of $\amalg(2849 \mathrm{~A} 1 / \mathbb{Q})$ is visible in $J_{0}(2849)$. Similarly, 4343B1 and 5389A1 have $\amalg$ of order 9 but degree not divisible by 3 .

But for all other cases examined, $\amalg(E / \mathbb{Q})\left[m_{E}\right]=$ $\amalg(E / \mathbb{Q})$ and we find much the same pattern as was exhibited by the examples given above, of conductors 571 and 681 . For convenience, we divide the results into, first, the cases where $\amalg$ has odd order greater than 1, and second, the cases of even order.

\section{The Shafarevich-Tate Groups of Odd Order}

For all but two of the optimal elliptic curve factors $E$ of squarefree conductor $N \leq 5500$ with $\amalg$ of odd order $p^{2}$, other than the "invisible" cases 2849A1, 4343B1 and 5389A1, we find another optimal elliptic curve factor $F$ which satisfies an $m$-congruence with $E$ and such that $F$ has trivial $\amalg$ but Mordell-Weil rank 2. The exceptions are 4229A1 (which is the only optimal curve of conductor 4229) and 5073D1 (where none of the other optimal curves of conductor 5073 has rank 2). A similar phenomenon occurs for all but four the curves $E$ whose conductor is in this range but is not squarefree, with $\amalg$ of order $p^{2}$. There are exceptions at levels 2392, 3364, 4914, and 5054 where we did not find any suitable congruent curve.

In most cases, $F$ has the same conductor as $E$, but for $E=3306 \mathrm{~B} 1$ and $E=5136 \mathrm{~B} 1$, which both have $\amalg$ of order 9 , the conductor of $F$ is a proper 
divisor of that of $E$ (and there is no suitable curve $F$ at the same level). The curve $E=3306 \mathrm{~B} 1$ satisfies a 3 -congruence with $F=1102 \mathrm{~A} 1$ which has rank 2 , and $E=5136 \mathrm{~B} 1$ is 3 -congruent to $F=1712 \mathrm{D} 1$ of rank 2 .

It would then follow that, with the exception of the exceptional cases listed above, all of $\amalg(E / \mathbb{Q})$ is visible in the abelian surface $J:=E \oplus F / \Phi$ where $\Phi \cong E[p] \cong F[p]$ and $J$ is a abelian subvariety of $J_{0}(M)$ for some $M$. Usually, $M=N$, and $J$ is even in the "new" part of $J_{0}(N)$, but there are exceptions to this as we have just seen.

In one case (conductor 2534) three optimal elliptic curve factors are all 3-congruent. Two of these elliptic curves (2534E1 and 2534F1) have Mordell-Weil rank zero and $\amalg$ of order 9 , and the third (2534G1) is the "explanatory" optimal factor: it has trivial U but Mordell-Weil rank equal to 2. The curve $4592 \mathrm{G} 1$ of rank 2 explains both the elements of order 5 in $4592 \mathrm{D} 1$, to which it is 5-congruent, and also the elements of order 3 in $4592 \mathrm{~F}$, to which it is 3 -congruent.

There is only one example here where $\amalg$ has order 49, namely 3364C1. However, this curve satisfies no congruence modulo 7 to any curve in the range studied, though its degree is a multiple of 7 , and neither of the other two curves at that level has rank 2. (These curves are the 29-twists of the curves 116ABC listed in [Cremona 1997], and all have rank 0.)

\section{The "Invisible" Examples}

Since 2849A1 is our first invisible example, it may be worth looking a bit more closely at it. Loïc Merel and Richard Taylor have suggested that one test to see if its Shafarevich-Tate group becomes visible in $J_{1}(2849)$. We have not yet made this test. The invisibility of this example in $J_{0}(2849)$ is the reason for the capitalization of the word "NONE" which appears in the " $F$-column" of its entry in the table. Similar remarks apply to the invisible examples 4343B1 and 5389A1.

\section{Examples Where $\Psi$ is of Even Order and E Has No Rational Point of Order 2}

Here a similar pattern is found. In Table 2, the congruences listed between curves with the same conductor are in most cases true modular 2-congruences proved using our first computational strategy. In a few such cases, and in all cases where the conductors are not equal, the first strategy failed and so we only claim that the curves "seem to" satisfy a congruence modulo 2, in the sense defined earlier. The exceptions, which are marked in the table, are: 3664J (for all three curves $F$ listed), $4528 \mathrm{C}$ and $4528 \mathrm{~A}$ (but the congruence between $4528 \mathrm{C}$ and $4528 \mathrm{~B}$ is proved), $4776 \mathrm{C}$ and $5296 \mathrm{C}$.

One feature peculiar to the prime $p=2$ is that it is possible for a "switch of parity" to occur; that is, it is possible for two optimal factors of $J_{0}(N)$ to admit a congruence modulo $p=2$ and have the property that they have different sign in their functional equations. Among the elliptic curves not possessing a rational point of order 2 , and of conductor at most 5500 with $\amalg$ of even order there are only two such cases which have a "parity switch". The first is $E=3431 \mathrm{~B} 1$ for which the 2-congruent curve $F$ has rank one. The order of $\amalg(E / \mathbb{Q})$ is $4 ; E$ admits a 2-congruence to both of the other optimal elliptic curves $3431 \mathrm{~A} 1$ and $3431 \mathrm{C} 1$ of its conductor, which both have rank 1 and no 2-torsion. Similarly, 3995A 1 has $\amalg$ of order 4 and is 2-congruent to $3995 \mathrm{D} 1$ which has rank 1 and no 2 -torsion. In the remaining cases where a corresponding $F$ exists, $F$ has Mordell-Weil rank 2.

There are cases where there is more than one congruent curve of rank 2 to explain the nontrivial elements of $\amalg$. At level 5302, there are two curves, 5302B1 and 5302J1, whose $\amalg$ has order 4 and 16 respectively, and which satisfy a congruence modulo 2 with each other and also with the four curves 5302C1-D1-F1-I1, all of which have rank 2.

As with the cases of odd order IU, there are several examples where we find a suitable explaining congruence with an optimal curve at a different level. For example, $\amalg(2045 B 1)$ is "explained" by curve 4090B1 of rank 2, to which 2045B1 "seems to be" 2-congruent.

\section{Examples Where $\amalg$ is of Even Order and E Has a Rational Point of Order Two}

There are 90 such elliptic curves E. All but three of these have $\amalg$ of order 4 and the remaining three, 2742B, 3800D, and 5335A, have $\amalg$ of order 16 . For all but eight of these 90 examples, there is another elliptic curve $F$ of the same conductor as $E$ 
which also possesses a rational point of order 2, and with positive Mordell-Weil rank. We have not yet checked which of these $82 \mathrm{~F}$ 's are (or even "seem to be") modular 2-congruent to their corresponding E's. The eight $E$ 's which do not possess a corresponding $F$ are 1105A, 2145D, 2145G, 3069A, 4901C, 5135B, 5185A, and 5335A.

\section{The Tables}

Tables 1 and 2 reproduce the data we have compiled. The 128 curves $E$ occurring in these tables comprise all optimal elliptic curves $E$ of conductor $N \leq 5500$ with nontrivial $\amalg$ except for the ninety optimal curves which have $\amalg$ of even order and a rational point of order 2. Each of these 128 elliptic curves $E$ is listed together with the corresponding elliptic curve $F$ of positive Mordell-Weil rank which "explains" $\amalg(E / \mathbb{Q})$ (except in the cases where $F$ doesn't exist). If there is no indication to the contrary, the congruence modulus linking $\amalg(E / \mathbb{Q})$ and $F$ is $\sqrt{|\amalg|}$. The modular degrees $m_{E}$ and $m_{F}$ are also tabulated: these were computed by the method of [Cremona 1995]. To save space, we do not give here the coefficients of a minimal Weierstrass equation for the curves; they may be obtained electronically [Cremona 1999].

\section{ASYMPTOTIC QUESTIONS}

We feel that these issues deserve to be investigated further. Is the prevalence of "visibility" a phenomenon occurring only in this modest range of conductors? Is most of $\amalg$ invisible? Or is most of II visible? It is relatively easy to find other examples where $\amalg(E / \mathbb{Q})$ is not annihilated by $m_{E}$ (and

\begin{tabular}{|c|c|c|c|c|c|c|c|c|c|}
\hline$E$ & $\sqrt{\left|\amalg_{E}\right|}$ & $m_{E}$ & $F$ & $m_{F}$ & $E$ & $\amalg_{E}$ & $m_{E}$ & $F$ & $m_{F}$ \\
\hline $681 B$ & 3 & $3 \cdot 5^{3}$ & $681 \mathrm{C}$ & $2^{5} \cdot 3$ & $3364 \mathrm{C}$ & 7 & $2^{6} \cdot 3^{2} \cdot 5^{2} \cdot 7$ & none & - \\
\hline $1058 \mathrm{D}$ & 5 & $2^{3} \cdot 3 \cdot 5 \cdot 7 \cdot 23$ & $1058 \mathrm{C}$ & $2^{4} \cdot 5$ & $3384 \mathrm{~A}$ & 5 & $2^{10} \cdot 3 \cdot 5 \cdot 11$ & $3384 \mathrm{C}$ & $2^{8} \cdot 5$ \\
\hline $1246 \mathrm{~B}$ & 5 & $2^{6} \cdot 3^{4} \cdot 5$ & $1246 \mathrm{C}$ & $2^{6} \cdot 5$ & $3536 \mathrm{H}$ & 3 & $2^{9} \cdot 3^{2} \cdot 5 \cdot 11$ & $3536 \mathrm{G}$ & $2^{7} \cdot 3^{2}$ \\
\hline $1664 \mathrm{~K}$ & 5 & $2^{7} \cdot 5 \cdot 7$ & $1664 \mathrm{~N}$ & $2^{6} \cdot 5$ & $3555 \mathrm{E}$ & 3 & $2^{3} \cdot 3 \cdot 5 \cdot 17$ & $3555 \mathrm{D}$ & $2^{7} \cdot 3 \cdot 5$ \\
\hline 1913B & 3 & $3 \cdot 103$ & $1913 \mathrm{~A}$ & $2^{2} \cdot 3 \cdot 5^{2}$ & $3712 \mathrm{~J}$ & 3 & $2^{6} \cdot 3 \cdot 13$ & $3712 \mathrm{I}$ & $2^{6} \cdot 3$ \\
\hline $2006 \mathrm{E}$ & 3 & $2^{6} \cdot 3 \cdot 5 \cdot 7 \cdot 23$ & $2006 \mathrm{D}$ & $2^{7} \cdot 3$ & $3879 \mathrm{E}$ & 3 & $2^{6} \cdot 3^{4} \cdot 5$ & 3879D & $2^{5} \cdot 3^{3}$ \\
\hline${ }^{1} 2366 \mathrm{D}$ & 3 & $2^{4} \cdot 3^{2} \cdot 13$ & $2366 \mathrm{E}$ & $2^{5} \cdot 3^{2} \cdot 5$ & $3933 \mathrm{~A}$ & 3 & $2^{5} \cdot 3 \cdot 5 \cdot 13$ & 3933B & $2^{6} \cdot 3 \cdot 5$ \\
\hline $2366 \mathrm{~F}$ & 5 & $2^{4} \cdot 3 \cdot 5 \cdot 13 \cdot 19$ & $2366 \mathrm{E}$ & $2^{5} \cdot 3^{2} \cdot 5$ & $3952 \mathrm{C}$ & 5 & $2^{4} \cdot 3 \cdot 5 \cdot 13 \cdot 17$ & $3952 \mathrm{E}$ & $2^{5} \cdot 3 \cdot 5$ \\
\hline $2429 \mathrm{~B}$ & 3 & $2 \cdot 3 \cdot 73$ & 2429D & $2^{3} \cdot 3 \cdot 13$ & $3954 \mathrm{C}$ & 3 & $2^{4} \cdot 3 \cdot 5^{3} \cdot 7^{2}$ & 3954D & $2^{5} \cdot 3 \cdot 5$ \\
\hline $2534 \mathrm{E}$ & 3 & $2^{2} \cdot 3^{2} \cdot 5^{3} \cdot 11$ & $2534 \mathrm{G}$ & $2^{5} \cdot 3^{2} \cdot 13$ & $4092 \mathrm{~A}$ & 5 & $2^{7} \cdot 3 \cdot 5 \cdot 19$ & 4092B & $2^{6} \cdot 3 \cdot 5$ \\
\hline $2534 \mathrm{~F}$ & 3 & $2^{2} \cdot 3^{2} \cdot 5 \cdot 7$ & $2534 \mathrm{G}$ & $2^{5} \cdot 3^{2} \cdot 13$ & $4229 \mathrm{~A}$ & 3 & $2^{3} \cdot 3 \cdot 7 \cdot 13$ & none & - \\
\hline $2541 \mathrm{D}$ & 3 & $2^{6} \cdot 3^{2} \cdot 7 \cdot 11$ & $2541 \mathrm{C}$ & $2^{5} \cdot 3^{2}$ & $4343 \mathrm{~B}$ & 3 & $2^{4} \cdot 1583$ & NONE & - \\
\hline $2574 \mathrm{D}$ & 5 & $2^{7} \cdot 3^{2} \cdot 5 \cdot 7^{2}$ & $2574 \mathrm{G}$ & $2^{8} \cdot 5$ & $4592 \mathrm{D}$ & 5 & $2^{8} \cdot 3^{2} \cdot 5 \cdot 17$ & $4592 \mathrm{G}$ & $2^{6} \cdot 3^{2} \cdot 5$ \\
\hline $2601 \mathrm{H}$ & 3 & $2^{8} \cdot 3 \cdot 17$ & $2601 \mathrm{~L}$ & $2^{8} \cdot 3$ & $4592 \mathrm{~F}$ & 3 & $2^{6} \cdot 3^{3} \cdot 7^{2}$ & $4592 \mathrm{C}$ & $2^{6} \cdot 3^{3}$ \\
\hline $2674 \mathrm{~B}$ & 3 & $2^{4} \cdot 3^{3} \cdot 13$ & $2674 \mathrm{~A}$ & $2^{4} \cdot 3^{2}$ & $4592 \mathrm{~F}$ & 3 & $2^{6} \cdot 3^{3} \cdot 7^{2}$ & $4592 \mathrm{G}$ & $2^{6} \cdot 3^{2} \cdot 5$ \\
\hline $2710 \mathrm{C}$ & 3 & $2^{5} \cdot 3^{3} \cdot 7$ & $2710 \mathrm{~B}$ & $2^{5} \cdot 3^{2}$ & $4606 \mathrm{~B}$ & 3 & $2^{8} \cdot 3^{3} \cdot 5 \cdot 7$ & $4606 \mathrm{C}$ & $2^{7} \cdot 3^{3}$ \\
\hline $2718 \mathrm{D}$ & 3 & $2^{6} \cdot 3 \cdot 5 \cdot 7 \cdot 29$ & $2718 \mathrm{~F}$ & $2^{6} \cdot 3 \cdot 5$ & $4675 \mathrm{~J}$ & 3 & $2^{2} \cdot 3^{3} \cdot 5^{3}$ & $4675 \mathrm{I}$ & $2^{6} \cdot 3^{3}$ \\
\hline $2768 \mathrm{C}$ & 3 & $2^{2} \cdot 3 \cdot 41$ & $2768 \mathrm{~B}$ & $2^{5} \cdot 3 \cdot 7$ & ${ }^{1} 4914 \mathrm{~N}$ & 3 & $2^{4} \cdot 3^{5}$ & none & - \\
\hline $2834 \mathrm{D}$ & 5 & $2^{2} \cdot 3 \cdot 5 \cdot 109$ & $2834 \mathrm{C}$ & $2^{6} \cdot 3^{2} \cdot 5$ & $4963 \mathrm{C}$ & 3 & $2^{2} \cdot 3 \cdot 71$ & $4963 \mathrm{D}$ & $2^{9} \cdot 3$ \\
\hline $2849 \mathrm{~A}$ & 3 & $2^{5} \cdot 5 \cdot 61$ & NONE & - & $5046 \mathrm{H}$ & 3 & $2^{4} \cdot 3 \cdot 5^{2} \cdot 7$ & $5046 \mathrm{~J}$ & $2^{4} \cdot 3 \cdot 5 \cdot 11$ \\
\hline $2900 \mathrm{D}$ & 5 & $2^{5} \cdot 3^{4} \cdot 5$ & $2900 \mathrm{C}$ & $2^{6} \cdot 3 \cdot 5$ & ${ }^{3} 5054 \mathrm{C}$ & 3 & $2^{3} \cdot 3^{3} \cdot 11$ & none & - \\
\hline${ }^{2} 2932 \mathrm{~A}$ & 3 & $3 \cdot 277$ & 1466B & $2^{4} \cdot 5 \cdot 13$ & $5073 \mathrm{D}$ & 3 & $2^{5} \cdot 3 \cdot 5 \cdot 7 \cdot 23$ & none & - \\
\hline 2955B & 3 & $2^{3} \cdot 3^{5} \cdot 5$ & $2955 \mathrm{C}$ & $2^{6} \cdot 3^{3}$ & $5082 \mathrm{C}$ & 5 & $2^{4} \cdot 3^{2} \cdot 5 \cdot 7 \cdot 11 \cdot 13$ & $5082 \mathrm{D}$ & $2^{8} \cdot 3 \cdot 5$ \\
\hline $3054 \mathrm{~A}$ & 3 & $2 \cdot 3 \cdot 5^{2} \cdot 11$ & $3054 \mathrm{C}$ & $2^{4} \cdot 3 \cdot 5 \cdot 7$ & ${ }^{2} 5136 \mathrm{~B}$ & 3 & $2^{4} \cdot 3 \cdot 59$ & $1712 \mathrm{D}$ & $2^{5} \cdot 7$ \\
\hline $3185 \mathrm{C}$ & 5 & $2^{4} \cdot 3 \cdot 5 \cdot 7 \cdot 11^{2}$ & $3185 \mathrm{~B}$ & $2^{4} \cdot 3 \cdot 5$ & $5389 \mathrm{~A}$ & 3 & $2^{2} \cdot 2333$ & NONE & - \\
\hline${ }^{2} 3306 \mathrm{~B}$ & 3 & $2^{4} \cdot 3^{3} \cdot 5^{2}$ & $1102 \mathrm{~A}$ & $2^{5} \cdot 3^{2}$ & $5499 \mathrm{E}$ & 3 & $2^{7} \cdot 3^{4} \cdot 5$ & $5499 \mathrm{~F}$ & $2^{7} \cdot 3^{3}$ \\
\hline
\end{tabular}

TABLE 1. Curves with odd $\left|\amalg_{E}\right|>1$, for all $N \leq 5500$. Notes keyed to the superscripted numbers to the left of the first column: ${ }^{1} E$ has rational 3-torsion. ${ }^{2}$ Curve $F$ is congruent to curve $E$ and has rank 2, but has a different level. If there is more than one such curve $F$, all are listed (on separate lines). ${ }^{3}$ The curve $5054 \mathrm{C}$ is the $(-19)$-twist of the curve $14 \mathrm{~A}$; it has a rational 3-isogeny but no rational torsion. 


\begin{tabular}{|c|c|c|c|c|c|c|c|c|c|}
\hline$E$ & $\sqrt{\left|\amalg_{E}\right|}$ & $m_{E}$ & $F$ & $m_{F}$ & $E$ & $\sqrt{\left|\amalg_{E}\right|}$ & $m_{E}$ & $F$ & $m_{F}$ \\
\hline $571 \mathrm{~A}$ & 2 & $2^{3} \cdot 3 \cdot 5$ & $571 \mathrm{~B}$ & $2^{4} \cdot 3$ & $54528 \mathrm{C}$ & 2 & $2^{7} \cdot 3$ & $4528 \mathrm{~A}$ & $2^{6} \cdot 5$ \\
\hline 1058B & 2 & $2^{4} \cdot 5 \cdot 23$ & $1058 \mathrm{C}$ & $2^{4} \cdot 5$ & $4528 \mathrm{C}$ & 2 & $2^{7} \cdot 3$ & $4528 \mathrm{~B}$ & $2^{6} \cdot 3$ \\
\hline${ }^{1} 1309 \mathrm{~A}$ & 4 & $2^{7} \cdot 3^{2} \cdot 17$ & 1309B & $2^{8}$ & ${ }^{1} 4544 \mathrm{M}$ & 2 & $2^{8} \cdot 3^{5}$ & $4544 \mathrm{~L}$ & $2^{8} \cdot 5$ \\
\hline $1325 \mathrm{D}$ & 2 & $2^{3} \cdot 3^{3} \cdot 5$ & $1325 \mathrm{E}$ & $2^{3} \cdot 3^{3}$ & $4544 \mathrm{M}$ & 2 & $2^{8} \cdot 3^{5}$ & $4544 \mathrm{G}$ & $2^{7} \cdot 5$ \\
\hline 1613B & 2 & $2^{4} \cdot 19$ & $1613 \mathrm{~A}$ & $2^{4} \cdot 5$ & $4564 \mathrm{C}$ & 2 & $2^{4} \cdot 3^{2} \cdot 5^{2}$ & $4564 \mathrm{~A}$ & $2^{4} \cdot 3 \cdot 11$ \\
\hline${ }^{1} 1701 \mathrm{I}$ & 2 & $2^{4} \cdot 3^{4}$ & $1701 \mathrm{~J}$ & $2^{4} \cdot 3^{3}$ & $4617 \mathrm{~F}$ & 2 & $2^{4} \cdot 3^{4}$ & $4617 \mathrm{H}$ & $2^{4} \cdot 3^{3}$ \\
\hline $1717 \mathrm{~A}$ & 2 & $2^{3} \cdot 41$ & 1717B & $2^{3} \cdot 13$ & $4630 \mathrm{~A}$ & 2 & $2^{9} \cdot 3 \cdot 5$ & 4630B & $2^{6} \cdot 3^{2}$ \\
\hline${ }^{1} 1738 \mathrm{~B}$ & 2 & $2^{11} \cdot 3^{3} \cdot 7$ & $1738 \mathrm{~A}$ & $2^{8}$ & $4630 \mathrm{~A}$ & 2 & $2^{9} \cdot 3 \cdot 5$ & $4630 \mathrm{C}$ & $2^{7} \cdot 3^{2}$ \\
\hline 1849D & 2 & $2^{4} \cdot 3 \cdot 7 \cdot 11$ & $1849 \mathrm{~A}$ & $2^{3} \cdot 3 \cdot 11$ & $4630 \mathrm{D}$ & 2 & $2^{6} \cdot 3 \cdot 5 \cdot 13$ & 4630B & $2^{6} \cdot 3^{2}$ \\
\hline${ }^{1} 1856 \mathrm{G}$ & 2 & $2^{8} \cdot 3 \cdot 5$ & $1856 \mathrm{D}$ & $2^{8}$ & 4630D & 2 & $2^{6} \cdot 3 \cdot 5 \cdot 13$ & $4630 \mathrm{C}$ & $2^{7} \cdot 3^{2}$ \\
\hline $1862 \mathrm{C}$ & 2 & $2^{4} \cdot 3^{3} \cdot 7$ & $1862 \mathrm{~A}$ & $2^{4} \cdot 3^{3}$ & ${ }^{1} 4655 \mathrm{G}$ & 2 & $2^{5} \cdot 3 \cdot 5 \cdot 7 \cdot 19$ & $4655 \mathrm{~F}$ & $2^{5} \cdot 3 \cdot 5$ \\
\hline 1888B & 2 & $2^{8} \cdot 3$ & $1888 \mathrm{~A}$ & $2^{7}$ & $4655 \mathrm{G}$ & 2 & $2^{5} \cdot 3 \cdot 5 \cdot 7 \cdot 19$ & $4655 \mathrm{C}$ & $2^{5} \cdot 3^{3} \cdot 7$ \\
\hline $1917 \mathrm{E}$ & 2 & $2^{3} \cdot 3^{4}$ & $1917 \mathrm{C}$ & $2^{3} \cdot 3^{3}$ & $4749 \mathrm{~A}$ & 2 & $2^{3} \cdot 3 \cdot 19 \cdot 23$ & 4749B & $2^{3} \cdot 7 \cdot 23$ \\
\hline $2023 \mathrm{~A}$ & 2 & $2^{4} \cdot 3^{3} \cdot 17$ & 2023B & $2^{4} \cdot 3^{3}$ & $4761 \mathrm{~A}$ & 2 & $2^{6} \cdot 5 \cdot 23$ & 4761B & $2^{6} \cdot 5$ \\
\hline $22045 B$ & 4 & $2^{3} \cdot 3 \cdot 5 \cdot 7 \cdot 17$ & $2045 \mathrm{C}$ & $2^{3} \cdot 3^{3} \cdot 13$ & ${ }^{5} 4776 \mathrm{C}$ & 2 & $2^{6} \cdot 3^{2} \cdot 5 \cdot 11$ & $4776 \mathrm{~B}$ & $2^{5} \cdot 5^{2}$ \\
\hline $32045 B$ & 4 & $2^{3} \cdot 3 \cdot 5 \cdot 7 \cdot 17$ & 4090B & $2^{6} \cdot 7$ & $4878 \mathrm{~A}$ & 2 & $2^{5} \cdot 17 \cdot 79$ & $4878 \mathrm{C}$ & $2^{6} \cdot 19$ \\
\hline 2089D & 2 & $2^{5} \cdot 3 \cdot 5$ & $2089 \mathrm{E}$ & $2^{5} \cdot 11$ & 4941B & 2 & $2^{3} \cdot 3^{2} \cdot 11$ & $4941 \mathrm{C}$ & $2^{3} \cdot 3^{4}$ \\
\hline${ }^{1} 2224 \mathrm{E}$ & 2 & $2^{7} \cdot 17$ & $2224 \mathrm{~F}$ & $2^{7} \cdot 3$ & $4975 \mathrm{C}$ & 2 & $2^{6} \cdot 5 \cdot 17$ & 4975B & $2^{6} \cdot 3^{3}$ \\
\hline${ }^{1} 2265 \mathrm{~A}$ & 2 & $2^{5} \cdot 3^{2} \cdot 5^{2} \cdot 7$ & $2265 B$ & $2^{5} \cdot 5 \cdot 7$ & $4975 \mathrm{C}$ & 2 & $2^{6} \cdot 5 \cdot 17$ & $4975 \mathrm{D}$ & $2^{6} \cdot 17$ \\
\hline 2409B & 2 & $2^{9} \cdot 5^{2}$ & 2409D & $2^{5} \cdot 7^{2}$ & $5046 \mathrm{C}$ & 2 & $2^{4} \cdot 3 \cdot 5^{2} \cdot 7 \cdot 29$ & $5046 \mathrm{~J}$ & $2^{4} \cdot 3 \cdot 5 \cdot 11$ \\
\hline $2541 \mathrm{~A}$ & 2 & $2^{5} \cdot 3^{4} \cdot 11$ & $2541 \mathrm{C}$ & $2^{5} \cdot 3^{2}$ & ${ }^{1} 5049 \mathrm{~A}$ & 2 & $2^{6} \cdot 3^{3} \cdot 5$ & 5049B & $2^{6} \cdot 3 \cdot 5^{2}$ \\
\hline 2554B & 2 & $2^{5} \cdot 13$ & $2554 \mathrm{C}$ & $2^{4} \cdot 3^{2} \cdot 7$ & ${ }^{3} 5067 \mathrm{C}$ & 2 & $2^{3} \cdot 3 \cdot 5 \cdot 13$ & $563 \mathrm{~A}$ & $2^{2} \cdot 13$ \\
\hline $2563 \mathrm{C}$ & 2 & $2^{6} \cdot 3 \cdot 7$ & $2563 \mathrm{D}$ & $2^{4} \cdot 3 \cdot 5$ & ${ }^{3} 5067 \mathrm{C}$ & 2 & $2^{3} \cdot 3 \cdot 5 \cdot 13$ & $1126 \mathrm{~A}$ & $2^{4} \cdot 11$ \\
\hline $2619 \mathrm{C}$ & 2 & $2^{4} \cdot 3^{2} \cdot 5$ & 2619D & $2^{4} \cdot 3 \cdot 5$ & ${ }^{3} 5067 \mathrm{C}$ & 2 & $2^{3} \cdot 3 \cdot 5 \cdot 13$ & $4504 \mathrm{~A}$ & $2^{6} \cdot 5$ \\
\hline $2678 \mathrm{~A}$ & 4 & $2^{9} \cdot 3^{2} \cdot 23$ & $2678 \mathrm{~B}$ & $2^{7} \cdot 3$ & $35067 \mathrm{C}$ & 2 & $2^{3} \cdot 3 \cdot 5 \cdot 13$ & 4504B & $2^{5} \cdot 13$ \\
\hline${ }^{2} 2678 \mathrm{~A}$ & 4 & $2^{9} \cdot 3^{2} \cdot 23$ & $2678 \mathrm{I}$ & $2^{5} \cdot 3 \cdot 11$ & ${ }^{3} 5067 \mathrm{C}$ & 2 & $2^{3} \cdot 3 \cdot 5 \cdot 13$ & $4504 \mathrm{C}$ & $2^{5} \cdot 17$ \\
\hline $2710 \mathrm{~A}$ & 2 & $2^{5} \cdot 3 \cdot 5^{2}$ & $2710 \mathrm{~B}$ & $2^{5} \cdot 3^{2}$ & ${ }^{2} 5117 \mathrm{C}$ & 4 & $2^{6} \cdot 3 \cdot 7 \cdot 37$ & 5117D & $2^{6} \cdot 5$ \\
\hline $2710 \mathrm{~A}$ & 2 & $2^{5} \cdot 3 \cdot 5^{2}$ & 2710D & $2^{5} \cdot 5 \cdot 11$ & $5133 \mathrm{C}$ & 2 & $2^{5} \cdot 31$ & 5133B & $2^{5} \cdot 3 \cdot 7$ \\
\hline $2738 \mathrm{C}$ & 4 & $2^{6} \cdot 3^{2} \cdot 37$ & $2738 \mathrm{D}$ & $2^{6} \cdot 3^{2}$ & $5133 \mathrm{C}$ & 2 & $2^{5} \cdot 31$ & 5133D & $2^{7} \cdot 5 \cdot 11$ \\
\hline $3017 \mathrm{~A}$ & 2 & $2^{3} \cdot 3^{5}$ & none & & $5150 \mathrm{C}$ & 2 & $2^{4} \cdot 3^{4} \cdot 5^{2}$ & $5150 \mathrm{D}$ & $2^{4} \cdot 3^{2} \cdot 5^{2}$ \\
\hline${ }^{1} 3370 \mathrm{D}$ & 2 & $2^{5} \cdot 5 \cdot 7$ & $3370 \mathrm{E}$ & $2^{5} \cdot 3^{4}$ & ${ }^{1} 5244 \mathrm{~A}$ & 2 & $2^{7} \cdot 3^{2} \cdot 5 \cdot 7$ & $5244 B$ & $2^{7} \cdot 3^{3}$ \\
\hline${ }^{1} 3380 \mathrm{~A}$ & 2 & $2^{6} \cdot 3^{3} \cdot 13$ & $3380 \mathrm{D}$ & $2^{6} \cdot 3^{2}$ & ${ }^{5} 5296 \mathrm{C}$ & 2 & $2^{4} \cdot 3 \cdot 37$ & 5296B & $2^{7} \cdot 3$ \\
\hline${ }^{4} 3431 \mathrm{~B}$ & 2 & $2^{3} \cdot 3^{3} \cdot 5$ & none & - & $5300 \mathrm{C}$ & 2 & $2^{4} \cdot 3^{2} \cdot 5 \cdot 23$ & $5300 \mathrm{G}$ & $2^{4} \cdot 3^{2} \cdot 23$ \\
\hline 3479D & 2 & $2^{6} \cdot 7 \cdot 13$ & $3479 \mathrm{E}$ & $2^{6} \cdot 13$ & $5302 \mathrm{~B}$ & 2 & $2^{5} \cdot 3 \cdot 5^{2}$ & $5302 \mathrm{C}$ & $2^{7} \cdot 5$ \\
\hline 3509B & 2 & $2^{4} \cdot 3^{2} \cdot 11^{2}$ & $3509 \mathrm{~A}$ & $2^{4} \cdot 3 \cdot 5$ & $5302 \mathrm{~B}$ & 2 & $2^{5} \cdot 3 \cdot 5^{2}$ & $5302 \mathrm{D}$ & $2^{6} \cdot 3^{2}$ \\
\hline $3555 \mathrm{C}$ & 2 & $2^{7} \cdot 3^{3} \cdot 5 \cdot 11$ & $3555 \mathrm{D}$ & $2^{7} \cdot 3 \cdot 5$ & $5302 \mathrm{~B}$ & 2 & $2^{5} \cdot 3 \cdot 5^{2}$ & $5302 \mathrm{~F}$ & $2^{8} \cdot 13$ \\
\hline $3575 \mathrm{E}$ & 2 & $2^{4} \cdot 3 \cdot 5^{2} \cdot 7$ & $3575 \mathrm{~F}$ & $2^{4} \cdot 3 \cdot 5 \cdot 7$ & $5302 \mathrm{~B}$ & 2 & $2^{5} \cdot 3 \cdot 5^{2}$ & $5302 \mathrm{I}$ & $2^{6} \cdot 5^{2}$ \\
\hline 5 3664J & 2 & $2^{4} \cdot 3^{2} \cdot 239$ & $3664 \mathrm{D}$ & $2^{6} \cdot 5$ & ${ }^{2} 5302 \mathrm{~J}$ & 4 & $2^{6} \cdot 101$ & $5302 \mathrm{C}$ & $2^{7} \cdot 5$ \\
\hline 5 3664J & 2 & $2^{4} \cdot 3^{2} \cdot 239$ & $3664 \mathrm{E}$ & $2^{6} \cdot 13$ & ${ }^{2} 5302 \mathrm{~J}$ & 4 & $2^{6} \cdot 101$ & $5302 \mathrm{D}$ & $2^{6} \cdot 3^{2}$ \\
\hline${ }^{5} 3664 \mathrm{~J}$ & 2 & $2^{4} \cdot 3^{2} \cdot 239$ & $3664 \mathrm{G}$ & $2^{9}$ & ${ }^{2} 5302 \mathrm{~J}$ & 4 & $2^{6} \cdot 101$ & $5302 \mathrm{~F}$ & $2^{8} \cdot 13$ \\
\hline $3686 \mathrm{D}$ & 4 & $2^{10} \cdot 3 \cdot 7^{2}$ & $3686 \mathrm{E}$ & $2^{11}$ & $5302 \mathrm{~J}$ & 4 & $2^{6} \cdot 101$ & $5302 \mathrm{I}$ & $2^{6} \cdot 5^{2}$ \\
\hline $3718 \mathrm{H}$ & 4 & $2^{8} \cdot 3 \cdot 5 \cdot 7 \cdot 13$ & $3718 \mathrm{~K}$ & $2^{8} \cdot 3$ & $5312 \mathrm{~K}$ & 2 & $2^{8} \cdot 3 \cdot 5$ & $5312 \mathrm{~F}$ & $2^{9}$ \\
\hline $3742 \mathrm{~A}$ & 2 & $2^{4} \cdot 3^{2} \cdot 5$ & $3742 \mathrm{~B}$ & $2^{4} \cdot 5 \cdot 7$ & ${ }^{1} 5312 \mathrm{~K}$ & 2 & $2^{8} \cdot 3 \cdot 5$ & $5312 \mathrm{~J}$ & $2^{8} \cdot 3$ \\
\hline${ }^{1} 3774 \mathrm{G}$ & 2 & $2^{10} \cdot 5 \cdot 7$ & $3774 \mathrm{D}$ & $2^{10} \cdot 3$ & $5390 \mathrm{E}$ & 2 & $2^{5} \cdot 3 \cdot 5 \cdot 7 \cdot 19$ & $5390 \mathrm{~L}$ & $2^{5} \cdot 3 \cdot 5 \cdot 19$ \\
\hline 3883B & 2 & $2^{3} \cdot 3^{3} \cdot 37$ & $3883 \mathrm{~A}$ & $2^{3} \cdot 3 \cdot 7$ & $5427 \mathrm{~A}$ & 2 & $2^{7} \cdot 3^{2}$ & 5427B & $2^{7} \cdot 3^{2}$ \\
\hline 3886B & 2 & $2^{6} \cdot 3 \cdot 5$ & $3886 \mathrm{G}$ & $2^{5} \cdot 3^{3}$ & $5427 \mathrm{~A}$ & 2 & $2^{7} \cdot 3^{2}$ & $5427 \mathrm{~F}$ & $2^{6} \cdot 3^{2}$ \\
\hline 3975B & 2 & $2^{5} \cdot 3 \cdot 7 \cdot 17$ & $3975 \mathrm{E}$ & $2^{5} \cdot 3 \cdot 5^{2}$ & $5427 \mathrm{E}$ & 2 & $2^{6} \cdot 3^{3}$ & 5427B & $2^{7} \cdot 3^{2}$ \\
\hline${ }^{6} 3995 \mathrm{~A}$ & 2 & $2^{6} \cdot 5 \cdot 7 \cdot 653$ & none & - & $5427 \mathrm{E}$ & 2 & $2^{6} \cdot 3^{3}$ & $5427 \mathrm{~F}$ & $2^{6} \cdot 3^{2}$ \\
\hline $4046 \mathrm{~F}$ & 2 & $2^{6} \cdot 3^{2} \cdot 7 \cdot 17$ & 4046D & $2^{6} \cdot 3^{2} \cdot 7$ & $5445 \mathrm{~A}$ & 2 & $2^{6} \cdot 3 \cdot 5 \cdot 11$ & $5445 B$ & $2^{6} \cdot 3 \cdot 5$ \\
\hline $4396 \mathrm{~A}$ & 2 & $2^{3} \cdot 3 \cdot 97$ & $4396 \mathrm{C}$ & $2^{3} \cdot 3^{4}$ & ${ }^{3} 5456 \mathrm{~A}$ & 2 & $2^{6} \cdot 3 \cdot 5 \cdot 19$ & $2728 \mathrm{C}$ & $2^{5} \cdot 3 \cdot 11$ \\
\hline $4428 \mathrm{~F}$ & 2 & $2^{3} \cdot 3^{5}$ & 4428B & $2^{3} \cdot 3^{4}$ & $35456 \mathrm{~A}$ & 2 & $2^{6} \cdot 3 \cdot 5 \cdot 19$ & $2728 \mathrm{D}$ & $2^{5} \cdot 11$ \\
\hline
\end{tabular}

TABLE 2. Curves with even $\left|\amalg_{E}\right|>1$, for all $N \leq 5500$. Notes keyed to the superscripted numbers to the left of the first column: ${ }^{1}$ Congruent modulo 4. ${ }^{2}$ Congruent modulo 2. ${ }^{3}$ Curve $F$ is congruent to curve $E$ and has rank 2 , but has a different level. If there is more than one such curve $F$, all are listed (on separate lines). ${ }^{4}$ The curve $3431 \mathrm{~B} 1$ is 2 -congruent to both $3431 \mathrm{~A} 1$ and $3431 \mathrm{C} 1$, which have rank $1 .{ }^{5}$ For these pairs, as well as all those for which $E$ and $F$ have different conductors, we only claim that $E$ and $F$ "seem to" satisfy a 2-congruence. 6 The curve $3995 \mathrm{~A} 1$ is 2 -congruent to $3995 \mathrm{D} 1$, which has rank 1. 
hence examples of invisible elements of $\amalg(E / \mathbb{Q})$ in $J_{0}(N)$ where $N$ is the conductor of $E$ ), if one searches among all twists (e.g., by quadratic Dirichlet characters) of a given modular elliptic curve.

To discuss asymptotics more specifically, if we are given a non-negative function $f(E)$ where $E$ ranges through all, or a class of, (modular) elliptic curves defined over $\mathbb{Q}$, let us define the upper conductor exponent of $f$ to be the minimal real number $\alpha$ having the property that for all $\varepsilon>0$ there is a finite $N(\varepsilon)$ such that

$$
f(E)<N^{\alpha+\varepsilon}
$$

if $\operatorname{conductor}(E)=N \geq N(\varepsilon)$ (putting $\alpha=\infty$ if there is no such real number). Thus, as Ram Murty [1999] has shown, the ABC conjecture is equivalent to the statement that the upper conductor exponent of the modular degree $\left(f(E)=m_{E}\right)$ for semistable elliptic curves is at most 2 . See also current publications of A. Granville in this regard. Also, Goldfeld and Szpiro [1995] have conjectured that the upper conductor exponent of the order of the Shafarevich-Tate group $(f(E)=|\amalg(E / \mathbb{Q})|)$ is at most $\frac{1}{2}$. See also [de Weger 1998], where it is shown (conditional on the Birch-Swinnerton-Dyer conjecture and the Riemann hypothesis for RankinSelberg zeta functions associated to certain modular forms of weight $\frac{3}{2}$ ) that the upper conductor exponent of $f(E)=|\amalg(E / \mathbb{Q})|$ is at least $\frac{1}{2}$.

Problem. What are the upper conductor exponents of orders of $\left|\amalg(E / \mathbb{Q})^{\circ}\right|$ and of $\left|\amalg(E / \mathbb{Q})\left[m_{E}\right]\right|$ as $E$ ranges through all optimal elliptic curves over $\mathbb{Q}$ ? What are they (i.e., are they any different) when $E$ ranges through all semi-stable optimal elliptic curves over $\mathbb{Q}$ ?

If it turns out that these upper conductor exponents are small it would be especially interesting to understand why so much of $\amalg$ for conductors $\leq 5500$ is visible, and is already visible in abelian surfaces, as our data shows.

\section{ACKNOWLEDGEMENTS}

We are deeply grateful to A. Agashé, N. Elkies, J. de Jong, A. Logan, L. Merel, W. McCallum, C. O'Neil, N. Shepherd-Barron, W. Stein and R. Taylor for comments, computations, explanations of the classical literature, and conversation, regarding this topic.

Finally, both authors would like to extend their warmest best wishes in his retirement year to Bryan Birch, to whom they owe so much.

\section{ELECTRONIC AVAILABILITY}

Most of the data used in these investigations, including the coefficients of minimal equations of all the elliptic curves mentioned here, their modular degrees and traces of Frobenius, may be obtained electronically [Cremona 1999].

\section{REFERENCES}

[Agashé 1999] A. Agashé, "On invisible elements of the Tate-Shafarevich group", C. R. Acad. Sci. Paris Sér. I Math. 328:5 (1999), 369-374.

[An et al. 1999] S. Y. An, S. Hammond, S. Y. Kim, W. McCallum, D. Marshall, and A. Perlis, "On the Jacobian of a curve of genus one", preprint, 1999.

[Carlton 1998] D. Carlton, Moduli for pairs of elliptic curves with isomorphic $N$-torsion, Ph.D. thesis, Mass. Inst. of Technology, Cambridge, MA, 1998.

[Cremona 1993] J. E. Cremona, "The analytic order of Ш for modular elliptic curves", J. Théor. Nombres Bordeaux 5:1 (1993), 179-184.

[Cremona 1995] J. E. Cremona, "Computing the degree of the modular parametrization of a modular elliptic curve", Math. Comp. 64:211 (1995), 1235-1250.

[Cremona 1997] J. E. Cremona, Algorithms for modular elliptic curves, 2nd ed., Cambridge Univ. Press, Cambridge, 1997.

[Cremona 1998] J. E. Cremona, "mwrank, a program for 2-descent on elliptic curves over $\mathbb{Q}$ ", last major update 1998. See http://www.maths.nottingham.ac.uk/ personal/jec/ftp/progs.

[Cremona 1999] J. E. Cremona, "Modular elliptic curve data for conductors up to 5500", last updated 1999. See http://www.maths.nottingham.ac.uk/ personal/jec/ftp/data.

[Cremona 2000] J. E. Cremona, "Classical invariants and 2-descent on elliptic curves", J. Symbolic Comp. (2000). To appear.

[Fisher 2000] T. Fisher, On 5 and 7 descents on elliptic curves, Ph.D. thesis, Cambridge University, Cambridge, submitted 2000. 
[Goldfeld and Szpiro 1995] D. Goldfeld and L. Szpiro, "Bounds for the order of the Tate-Shafarevich group", Compositio Math. 97:1-2 (1995), 71-87. Special issue in honour of Frans Oort.

[Grothendieck 1968] A. Grothendieck, "Le groupe de Brauer, II: Théorie cohomologique", pp. 67-87 in Dix exposés sur la cohomologie des Schémas, edited by A. Grothendieck and N. H. Kuiper, Adv. Stud. Pure Math. 3, North-Holland, Amsterdam, 1968.

[Kani and Schanz 1997] E. Kani and W. Schanz, "Diagonal quotient surfaces", Manuscripta Math. 93:1 (1997), 67-108.

[Kani and Schanz 1998] E. Kani and W. Schanz, "Modular diagonal quotient surfaces", Math. Z. 227:2 (1998), 337-366.

[Mazur and Ribet 1991] B. Mazur and K. A. Ribet, "Two-dimensional representations in the arithmetic of modular curves", pp. 215-255 in Courbes modulaires et courbes de Shimura (Orsay, 1987/1988), Astérisque 196-197, 1991.

[Merriman et al. 1996] J. R. Merriman, S. Siksek, and N. P. Smart, "Explicit 4-descents on an elliptic curve", Acta Arith. 77:4 (1996), 385-404.

[Mumford 1966] D. Mumford, "On the equations defining abelian varieties, I", Invent. Math. 1 (1966), 287-354.

[Murty 1999] M. R. Murty, "Bounds for congruence primes", pp. 177-192 in Automorphic forms, automorphic representations, and arithmetic (Fort Worth, TX, 1996), edited by R. S. Doran et al., Proc. symp. pure math. 66, Amer. Math. Soc., Providence, RI, 1999.

[O'Neil 1999] C. H. O'Neil, Jacobians of curves of genus one, Ph.D. thesis, Harvard University, Cambridge, MA, 1999.
[Ribet 1990] K. A. Ribet, "On modular representations of $\operatorname{Gal}(\overline{\mathbb{Q}} / \mathbb{Q})$ arising from modular forms", Invent. Math. 100:2 (1990), 431-476.

[Salmon 1879] G. Salmon, A treatise on the higher plane curves, 3rd ed., Hodges, Foster and Figgis, Dublin, 1879. Reprinted by Chelsea, New York, 1960.

[Salmon 1928] G. Salmon, A treatise on the analytic geometry of three dimensions, edited by C. H. Rowe, London, 1928. Reprinted by Chelsea, New York, 1958.

[Stevens 1989] G. Stevens, "Stickelberger elements and modular parametrizations of elliptic curves", Invent. Math. 98:1 (1989), 75-106.

[Sturm 1987] J. Sturm, "On the congruence of modular forms", pp. 275-280 in Number theory (New York, 1984/1985), edited by D. V. Chudnovsky et al., Lecture Notes in Math. 1240, Springer, Berlin, 1987.

[Tate 1968] J. Tate, "On the conjectures of Birch and Swinnerton-Dyer and a geometric analog", pp. 415440, Exp. No. 306 in Séminaire Bourbaki 1965/66, New York, Benjamin, 1968. Reprinted as pp. 189-214 of Dix exposés sur la cohomologie des schémas, edited by A. Grothendieck and N. H. Kuiper, Adv. Stud. Pure Math. 3, North-Holland, Amsterdam, 1968; also in vol. 9 of the Sém. Bourbaki reprint by Soc. Math. France, Paris, 1995.

[de Weger 1998] B. M. M. de Weger, " $A+B=C$ and big U's", Quart. J. Math. Oxford Ser. (2) 49:193 (1998), $105-128$.

[Weil 1954] A. Weil, Remarques sur un memoire d'Hermite, vol. 5, 1954. Reprinted as pp. 111-116 of Euvres scientifiques / Collected papers, v. 2 (19511964), Springer, New York, 1979.

John E. Cremona, Department of Mathematics, University of Exeter, North Park Road, Exeter EX4 4QE, United Kingdom (cremona@maths.exeter.ac.uk)

Barry Mazur, Department of Mathematics, Harvard University, Cambridge, MA 02138, United States (mazur@math.harvard.edu)

Received October 14, 1998; accepted in revised form January 19, 1999 